DOI: $10.24850 / j-t y c a-2022-01-06$

Artículos

\title{
Modelos de evapotranspiración de referencia diaria en la zona arrocera del Valle del Cauca, Colombia
}

\section{Daily reference evapotranspiration models in the rice zone of Valle del Cauca, Colombia}

Jesica Natalia Valencia-Gonzalez ${ }^{1}$, ORCID: https://orcid.org/0000-00030807-2554

Ramón Arteaga-Ramírez², ORCID: http://orcid.org/0000-0001-94593588

Mario Alberto Vázquez-Peña ${ }^{3}$, ORCID: https://orcid.org/0000-00032084-7420

Abel Quevedo-Nolasco4, ORCID: https://orcid.org/0000-0003-33035077

1Universidad Autónoma Chapingo, Texcoco, Estado de México, México, nvg.912@gmail.com

²Universidad Autónoma Chapingo, Texcoco, Estado de México, México, rarteagar@taurus.chapingo.mx

33niversidad Autónoma Chapingo, Texcoco, Estado de México, México, mavazquez.coahuila@gmail.com 
${ }^{4}$ Colegio de Posgraduados, Texcoco, Estado de México, México, abcdqn@gmail.com

Autor para correspondencia: Ramón Arteaga-Ramírez, rarteagar@taurus.chapingo.mx

\section{Resumen}

Los cálculos oportunos de la evapotranspiración de referencia diaria (ETo) cuantifican y planifican el uso de la disponibilidad hídrica y, en consecuencia, determinan un consumo diario, más en las condiciones donde la falta de información climática interrumpe el rendimiento de los diversos modelos para estimar la evapotranspiración de referencia diaria en una determinada región. El objetivo de este trabajo es evaluar el comportamiento de las ecuaciones de Hargreaves-Samani, Priestley y Taylor, García y López, y Linacre en el cálculo de la evapotranspiración de referencia diaria (ETo) respecto a la ecuación de FAO Penman-Monteith (PM), utilizando la información diaria de cinco estaciones próximas a la zona productora de arroz del Valle del Cauca, Colombia. Se estimó la evapotranspiración de referencia diaria de 28 años consecutivos; se utilizaron los indicadores estadísticos: coeficiente de determinación $\left(R^{2}\right)$; la raíz cuadrada del cuadrado medio del error (RMSE); eficiencia de NashSutcliffe (NSE), y diagramas de cajas para definir la bondad de los modelos utilizados. Los modelos Priestley-Taylor (PT) y Linacre (LN) han exhibido los resultados más afines al modelo estándar. Las estaciones Ing. Bengala y Ciat Quilichao se exhiben como las áreas que mejores 
comportamientos poseen de todos los modelos con el índice del coeficiente de determinación $\left(R^{2}\right)$; todas las estaciones presentaron resultados aceptables con la raíz cuadrada del cuadrado medio del error (RMSE) para las diversas comparaciones realizadas, no así con la eficiencia de Nash-Sutcliffe. Existe evidencia limitada del uso diario del modelo García y López, siendo este método y el de Hargreaves-Samani los menos ideales para la estación Independencia.

Palabras clave: modelos de evapotranspiración, arroz, datos diarios, evapotranspiración de referencia.

\section{Abstract}

The opportune calculations of the daily reference evapotranspiration (ETo) quantify and schedule the use of the water availability and consequently determine a daily consumption, mostly in the conditions where the lack of climatic information interrupts the performance of diverse models to estimate the daily reference evapotranspiration in a certain region. Therefore, the objective of this work is to evaluate the behavior of the equations of Hargreaves-Samani, Priestley and Taylor, García and López, and Linacre in the calculation of daily reference evapotranspiration (ETo) for the equation of FAO Penman-Monteith (PM) using daily information from five stations near the rice production area of Valle del Cauca, Colombia. The daily evapotranspiration of 28 consecutive years was estimated, the results were confirmed by the statistical indicators: Determination Coefficient $\left(R^{2}\right)$; the square root of the mean 
square of the error (RMSE); Nash-Sutcliffe Efficiency (NSE), and box diagrams. The Priestley-Taylor (PT) and Linacre (LN) models have shown the closest results to the standard model. Bengala and Ciat Quilichao stations are shown as the areas with the best behavior of all the models with the coefficient of determination index $\left(R^{2}\right)$; all the stations presented acceptable results with the square root of the mean square of the error (RMSE) for the different comparisons made but not with the Nash-Sutcliffe Efficiency (Nash-Sutcliffe Efficiency). However, there is limited evidence of the daily use of the Garcia and Lopez model, being this method and that of the Hargreaves-Samani the least ideal for the Independence station.

Keywords: Evapotranspiration models, rice, daily data, reference evapotranspiration.

Recibido: 03/08/2020

Aceptado: $10 / 02 / 2021$

\section{Introducción}


Las estimaciones de evapotranspiración de referencia (ETo) han sido ampliamente estudiadas y utilizadas en los fundamentos de ingeniería de riego para definir las necesidades de agua en los cultivos. De acuerdo con Sánchez (2000), el término de evapotranspiración de referencia (ETo) ha sido usado para describir los procesos totales de trasferencia de agua a la atmósfera desde una vegetación con características bien definidas. En la agricultura, la ETo es una de las variables elementales para promover un uso más eficiente del agua, por esto la necesidad de tener estimaciones adecuadas (Back, 2008), ya que juegan un papel esencial en el balance hídrico para el manejo de cuencas y para la estimación de los requerimientos de riego, la programación del riego y estudios sobre el cambio climático.

El sur del Valle del Cauca-Colombia posee dos épocas de siembra a nivel secano con 3750 ha productoras de arroz (Dane \& Fedearroz, 2017); la cuantificación de la ETo diaria para la zona a nivel diario es necesaria para mejorar el manejo del cultivo, conocer sus necesidades hídricas y realizar un uso eficiente del agua (Martínez \& Pérez, 2006); pero para estimar dicha variable se carecen de registros diarios para utilizar el modelo Penman-Monteith (PM) propuesto por Allen, Pereira, Raes y Smith (1998). En Colombia existen algunos trabajos relacionados con la estimación de la ETo a diferentes escalas: Barco et al. (2000) concluyeron que Penman y Morton son métodos difíciles de implementar debido a la información climática requerida; la ecuación Cenicafe desarrollada por Jaramillo (2006) permite calcular la evapotranspiración 
Tecnología y

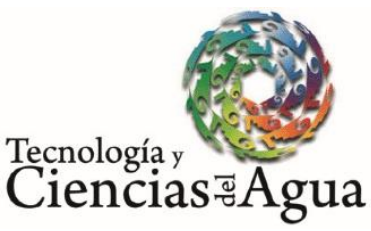

2022, Instituto Mexicano de Tecnología del Agua

Open Access bajo la licencia CC BY-NC-SA 4.0

(https://creativecommons.org/licenses/by-nc-sa/4.0/)

(ETo) de forma sencilla, dicha ecuación solo depende de la elevación sobre el nivel del mar; la ETo estimada para todo el país por Poveda et al. (2007) indica que las ecuaciones presentadas por Turc y Morton son las más convenientes para estas evaluaciones.

La tendencia de la estimación de este tipo de variable meteorológica ha indicado que se reportan más resultados obtenidos a escala anual y mensual; no obstante, se han identificado en Scopus algunos reportes significativos sobre la estimación de la evapotranspiración de referencia diaria utilizada en la estimación de la ET del cultivo del arroz publicados en 2017 en Taiwán y en 2019 en Senegal. Al recurrir a los ejemplos, Yao et al. (2017) obtuvieron los mapas de la ETo promedio de 10 días a partir de imágenes satelitales meteorológicas, comparada con la ETo derivada del modelo PM de la FAO (Food and Agriculture Organization of the United Nations) (Allen et al., 1998); Djaman, Sall, Sow, Manneh e Irmak (2019) determinaron los datos climáticos medidos en el área del arroz de riego que se utilizan en la estimación de la ETo mediante el modelo PenmanMonteith en condiciones en las que no existen registros de las estaciones meteorológicas.

La elección de un modelo o método de cálculo depende de la disponibilidad de los datos meteorológicos, así como de la exactitud y precisión del modelo para una región dada (De-Carvalho et al., 2013). Por ende, La FAO ha adoptado el modelo de Penman-Monteith principalmente por dos razones: a) se trata de un modelo físico aplicable no sólo a una zona en específico, y b) los datos que se usan se pueden obtener de estaciones meteorológicas, observatorios o mediciones a través de lisímetros (López, Hess, \& White, 2009). Expuesto todo lo 
anterior, existe para el valle del Cauca por lo menos un modelo que estime con un error bajo la ETo a nivel diario con respecto a un modelo estándar. El objetivo de este trabajo fue evaluar el comportamiento de las ecuaciones de Hargreaves-Samani, Priestley-Taylor, García y López, y Linacre en el cálculo de la evapotranspiración de referencia diaria (ETo) respecto a la ecuación de la FAO Penman-Monteith (PM).

\section{Materiales y métodos}

La zona arrocera al sur del Valle del Cauca se extiende aproximadamente entre $\operatorname{los} 3^{\circ} 5^{\prime} 0^{\prime \prime}$ a los $3^{\circ} 20^{\prime} 0^{\prime \prime}$ de latitud norte y entre los $76^{\circ} 10^{\prime} 0^{\prime \prime}$ y $76^{\circ} 50^{\prime} 0^{\prime \prime}$ de longitud oeste. La elevación de la zona es de unos 1000 msnm; predomina una temperatura media de $23^{\circ} \mathrm{C}$, con una oscilación diaria de $11{ }^{\circ} \mathrm{C}$; húmeda relativa del 73 al $80 \%$; brillo solar de 138 a 200 horas mensuales (Valencia, García, \& Montero, 2017). Según Köppen, corresponde al clima tropical lluvioso de bosque (Martínez \& Mendivelso, 2004: p. 53) (Figura 1). 


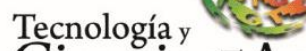

Ciencias $₫$ Agua
2022, Instituto Mexicano de Tecnología del Agua

Open Access bajo la licencia CC BY-NC-SA 4.0

(https://creativecommons.org/licenses/by-nc-sa/4.0/)

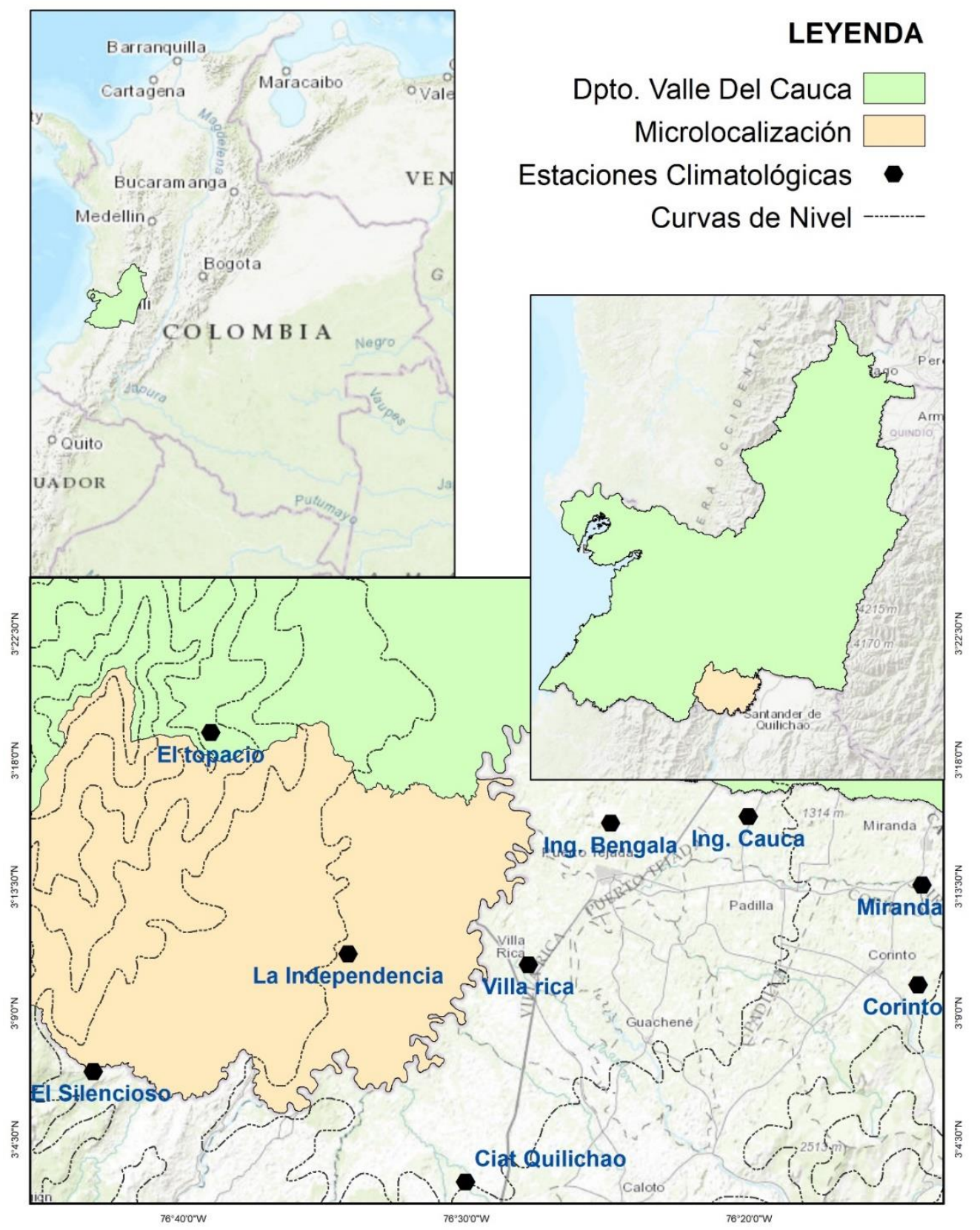

Figura 1. Localización del área de estudio. 
Se usaron los registros diarios de la temperatura máxima, temperatura mínima, humedad relativa y brillo solar de cinco estaciones climáticas por un periodo de estudio de 1989 a 2016 (Tabla 1).

Tabla 1. Descripción de las estaciones climáticas.

\begin{tabular}{|c|c|c|c|}
\hline Estación & $\begin{array}{c}\text { Altitud } \\
\mathbf{( m s n m )}\end{array}$ & Latitud (N) & Longitud (W) \\
\hline Ciat Quilichao & 977 & $03^{\circ} 03^{\prime}$ & $76^{\circ} 30^{\prime}$ \\
\hline Ingenio Bengala & 1000 & $03^{\circ} 15^{\prime}$ & $76^{\circ} 24^{\prime}$ \\
\hline Ingenio Cauca & 1000 & $03^{\circ} 14^{\prime}$ & $76^{\circ} 13^{\prime}$ \\
\hline La Independencia & 963 & $03^{\circ} 11^{\prime} 8^{\prime \prime}$ & $76^{\circ} 34^{\prime} 9^{\prime \prime}$ \\
\hline Ingenio Miranda & 1128 & $03^{\circ} 16^{\prime}$ & $76^{\circ} 20^{\prime}$ \\
\hline
\end{tabular}

\section{Modelos para estimar la evapotranspiración de referencia diaria (Eto)}

Se calculó la ETo con los cinco modelos reconocidos a nivel diario en el programa Excel. A continuación se enlistan los modelos. 


\section{Ecuación Penman Monteith (PM)}

El método Penman-Monteith es considerado el modelo estándary el más preciso para estimar ETo (Allen et al., 1998). Fue desarrollado con la definición del cultivo de referencia, que es cultivo hipotético de altura de $0.12 \mathrm{~m}$, con una resistencia superficial de $70 \mathrm{~s} \mathrm{~m}^{-1} \mathrm{y}$ un albedo de 0.23 , y que representa la evapotranspiración de una superficie extensa de pasto verde de altura uniforme, creciendo activa y adecuadamente regado (Allen, Pereira, Raes, \& Smith, 2006):

$E T o_{P M}=\frac{0.408 \triangle(R n-G)+\gamma * \frac{900}{T+273} u_{2}\left(e_{s}-e_{a}\right)}{\Delta+\gamma\left(1+0,34 u_{2}\right)}$

ETopm: evapotranspiración de referencia $\left(\mathrm{mm} \mathrm{día}^{-1}\right)$.

$\mathrm{Rn}$ : radiación neta en la superficie del cultivo $\left(\mathrm{MJ} \mathrm{m}^{-2} \mathrm{~d}^{-1}\right)$.

G: flujo de calor de suelo (MJ $\left.\mathrm{m}^{-2} \mathrm{~d}^{-1}\right)$.

$\mathrm{T}$ : temperatura media del aire a $2 \mathrm{~m}$ de altura $\left({ }^{\circ} \mathrm{C}\right)$.

$\mathrm{u}_{2}$ : velocidad del viento a $2 \mathrm{~m}$ de altura $\left(\mathrm{m} \mathrm{s}^{-1}\right)$.

$E_{s}-e_{a}$ : déficit de presión de vapor $(\mathrm{KPa})$.

$\Delta$ : pendiente de la curva de presión de vapor a saturación $\left(\mathrm{KPa}^{\circ} \mathrm{C}^{-1}\right)$. 
Tecnología y

Ciencias $₫$ Agua
2022, Instituto Mexicano de Tecnología del Agua

Open Access bajo la licencia CC BY-NC-SA 4.0

(https://creativecommons.org/licenses/by-nc-sa/4.0/)

$\gamma$ : constante psicrométrica $\left(\mathrm{Kpa}^{\circ} \mathrm{C}^{-1}\right)$.

El procesamiento de los datos por ser llevado a escala diaria el valor $\mathrm{G}=0$ para el cultivo de referencia es según Allen et al. (2006).

\section{Ecuación Hargreaves-Samani (HS)}

El modelo de Hargreaves-Samani es un modelo más simple que requiere de variables meteorológicas, temperatura (media, máxima y mínima), radiación incidente (Xu \& Singh, 2001) y radiación solar extraterrestre $\left(R_{a}\right)$ para estimar la ETo para una latitud y día determinados; $R_{a}$ puede obtenerse a partir de tablas o calcularse mediante un conjunto de ecuaciones que utilizan la temperatura:

ETo $_{H S}=0.0023\left(T_{\text {media }}+17.8\right)\left(T_{\text {max }}-T_{\text {min }}\right)^{0.5} R_{a}$

\section{Ecuación de Priestley-Taylor (PT)}


Es un modelo semiempírico que requiere de menos parámetros para el cálculo de la evapotranspiración, comparado con el modelo de PenmanMonteith (Cervantes, Arteaga, Vázquez, Ojeda, \& Quevedo, 2013). Esta metodología involucra la radiación solar y manifiesta menos errores respecto del método estándar (Pereira, 2004). Los elementos necesarios para su cálculo son:

$\Delta$ : pendiente de la curva de vapor de saturación $\left(\mathrm{KPa}^{\circ} \mathrm{C}^{-1}\right)$.

A: coeficiente empírico equivalente a 1.26.

$\gamma$ : constante psicrométrica $\left(\mathrm{KPa}^{\circ} \mathrm{C}^{-1}\right)$.

$R_{n}$ : radiación neta del cultivo $\left(\mathrm{MJ} \mathrm{m}^{-2} \mathrm{~d}^{-1}\right)$.

$G$ : flujo de calor de suelo (MJ $\mathrm{m}^{-2} \mathrm{~d}^{-1}$ ), por ser a escala diaria el valor $\mathrm{G}$ $=0$ para el cultivo de referencia según Allen et al. (2006):

$E T o_{P T}=\alpha *\left[\frac{\Delta}{\Delta+\gamma}\right] *(R n-G)$

\section{Ecuación de Linacre (LN)}


Este modelo se deriva de Penman, estimando la evapotranspiración a través de datos geográficos (latitud y altitud) y de temperatura (Carvalho, Rios, Miranda, \& Castro, 2011):

$E T o L N=\frac{\left(\frac{500 * T m}{100-A}\right)+15 *(T-T P R)}{80-T}$

EToLN, en mm día-1.

$\mathrm{Tm}=\mathrm{T}+0.006 * \mathrm{~h}$.

h: altura del lugar, en msnm.

TPR: temperatura del punto de rocío, ${ }^{\circ} \mathrm{C}$.

A: latitud del lugar (grados decimales).

$\mathrm{T}$ : temperatura media, ${ }^{\circ} \mathrm{C}$.

\section{Ecuación de García y López (GL)}

El modelo propuesto se adapta a las latitudes comprendidas desde los $15^{\circ}$ $\mathrm{N}$ hasta los $15^{\circ} \mathrm{S}$. Las variables utilizadas son la temperatura media en ${ }^{\circ} \mathrm{C}$ y la humedad relativa en \% (García \& López, 1970): 
EToGL $=\left[1.21 * 10^{\frac{7.45 * T}{234.7+T}} *(1-0.01 * H R)\right]+0.21 * T-2.3$

\section{Modelo de regresión lineal}

Se utilizaron cinco estaciones para definir los diagramas de dispersión y la relación que existe entre los datos calculados con el modelo de PM y los estimados con las ecuaciones de HS, PT, LN y GL, de las cuales se obtienen los siguientes modelos lineales:

$E T o_{P M-H S}=a+b * E T o H S$

$E T o_{P M-P T}=a+b * E T o P T$

$E T o_{P M-L N}=a+b * E T o L N$

$E T o_{P M-G L}=a+b * E T o G L$

\section{Índices estadísticos}


Los índices utilizados para evaluar la bondad de ajuste de los modelos son:

a) El coeficiente de determinación $\left(R^{2}\right)$ superior a 0.60 indica el grado en que la regresión explica la suma del total al cuadrado (Tagliaferre et al., 2012).

b) La raíz cuadrada del cuadrado medio del error compara un valor predicho y un valor observado o conocido (RMSE) (Caí, Liu, Lei, \& Santos, 2007).

c) Eficiencia de Nash-Sutcliffe (NSE): este coeficiente establece la relación entre el comportamiento real y el modelado. El dominio matemático del valor del coeficiente NSE es de $-\infty$ a 1 , donde la unidad representa la simulación perfecta. Un ajuste perfecto quiere decir que la varianza de los errores es cero; vale cero cuando la varianza de los errores es igual a la varianza observada, lo cual significa que el modelo produce estimaciones del promedio de las observaciones en todos los intervalos. Los valores negativos indican un desempeño malo del modelo (Magana-Hernández, Ba, Guerra-Cobian, \& Víctor, 2013).

\section{Diagrama de cajas}


Se emplearon estos gráficos para identificar la forma en que se distribuyen la media y desviación estándar, y la manera en que se distribuyen los datos calculados por todos los modelos para cada estación.

\section{Resultados}

Se presenta el comportamiento de la evapotranspiración de referencia de algunos años hidrológicos representativos para la zona productora evaluada. El año 1999 se eligió como el más Iluvioso (10 513 mm año-1 para la zona), 2012 fue un año intermedio (7 $791 \mathrm{~mm}$ año-1) y 1992 un año seco (5 $544 \mathrm{~mm}$ año-1).

De hecho, PT y GL presentan resultados similares en el año lluvioso; pero en el año intermedio y seco, GL presentó valores superiores a PT, pero inferiores a HS. Otra observación considerable es que el método de LN mostró estimaciones superiores en los meses de julio, agosto y septiembre, ya que la temperatura es superior en esta época del año e influye en la estimación por este método (Figura 2, Figura 3, Figura 4). 
Tecnología y

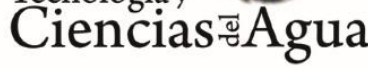

2022, Instituto Mexicano de Tecnología del Agua

Open Access bajo la licencia CCBY-NC-SA 4.0

(https://creativecommons.org/licenses/by-nc-sa/4.0/)

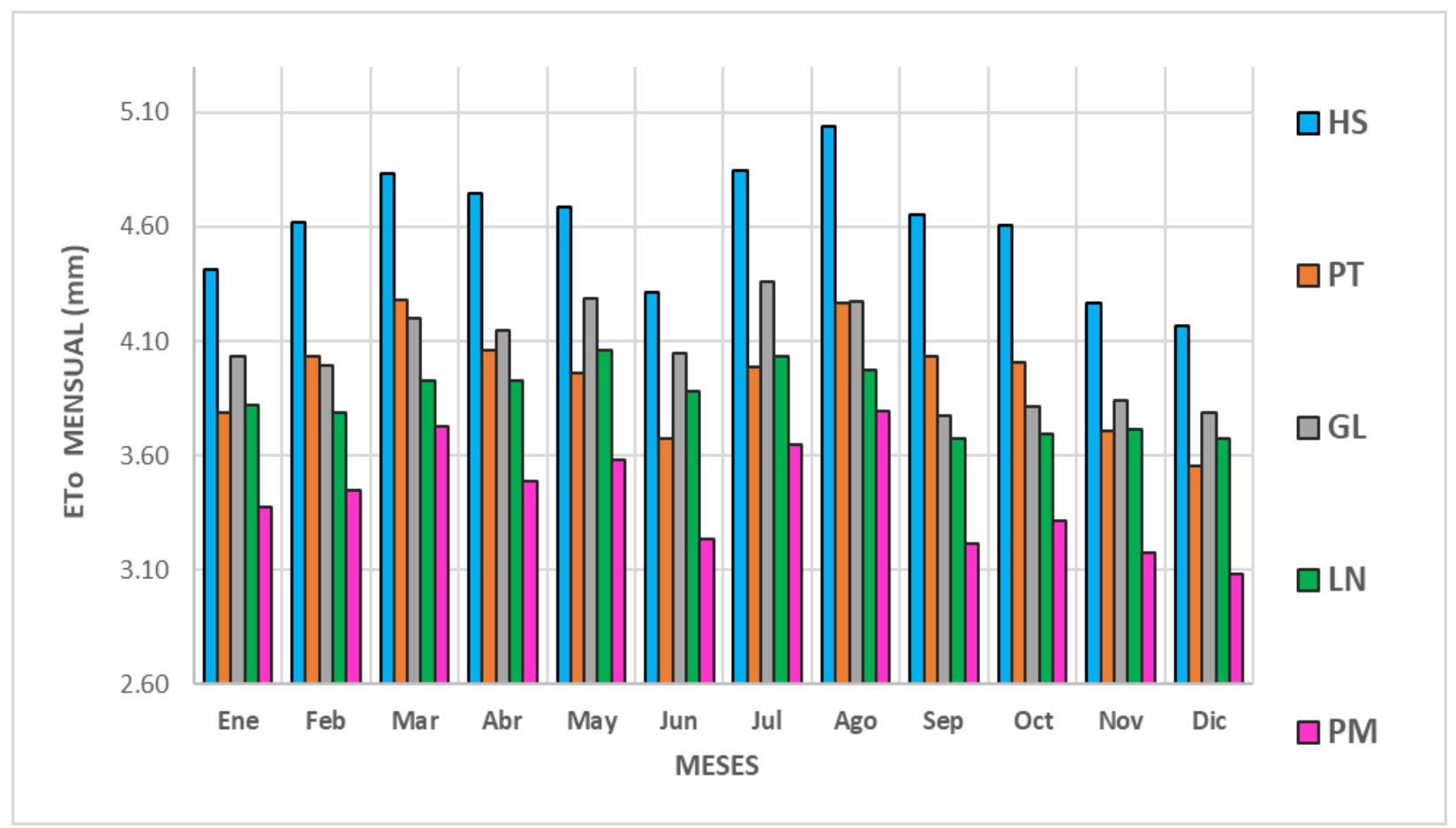

Figura 2. ETo promedio mensual para un año lluvioso en la zona productora de arroz. 
Tecnología y

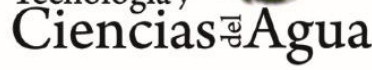

2022, Instituto Mexicano de Tecnología del Agua

Open Access bajo la licencia CCBY-NC-SA 4.0

(https://creativecommons.org/licenses/by-nc-sa/4.0/)

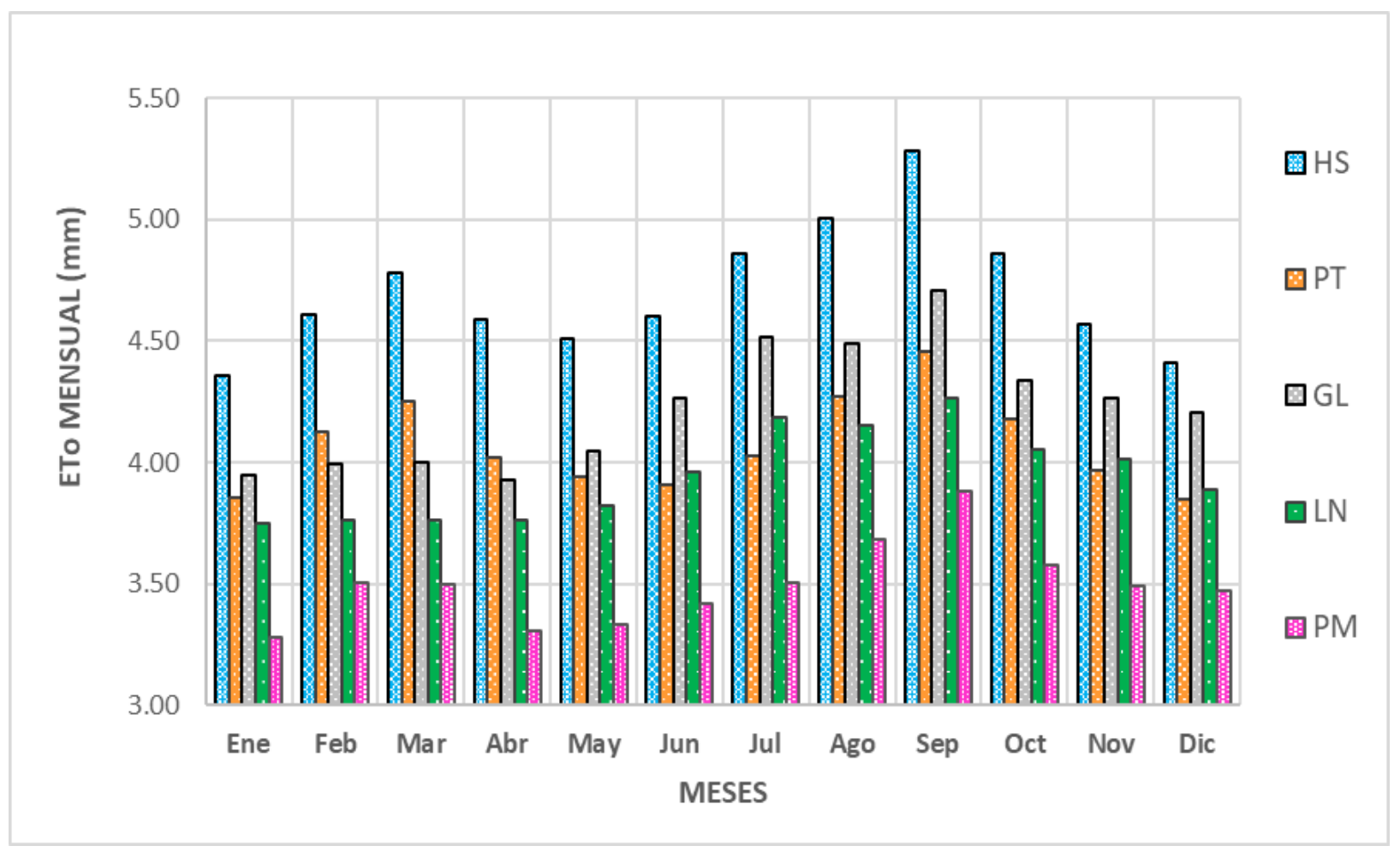

Figura 3. ETo promedio mensual para un año intermedio en la zona productora de arroz. 
Tecnología y

Ciencias $\stackrel{\Xi}{\Im}$ Agua
2022, Instituto Mexicano de Tecnología del Agua

Open Access bajo la licencia CC BY-NC-SA 4.0

(https://creativecommons.org/licenses/by-nc-sa/4.0/)

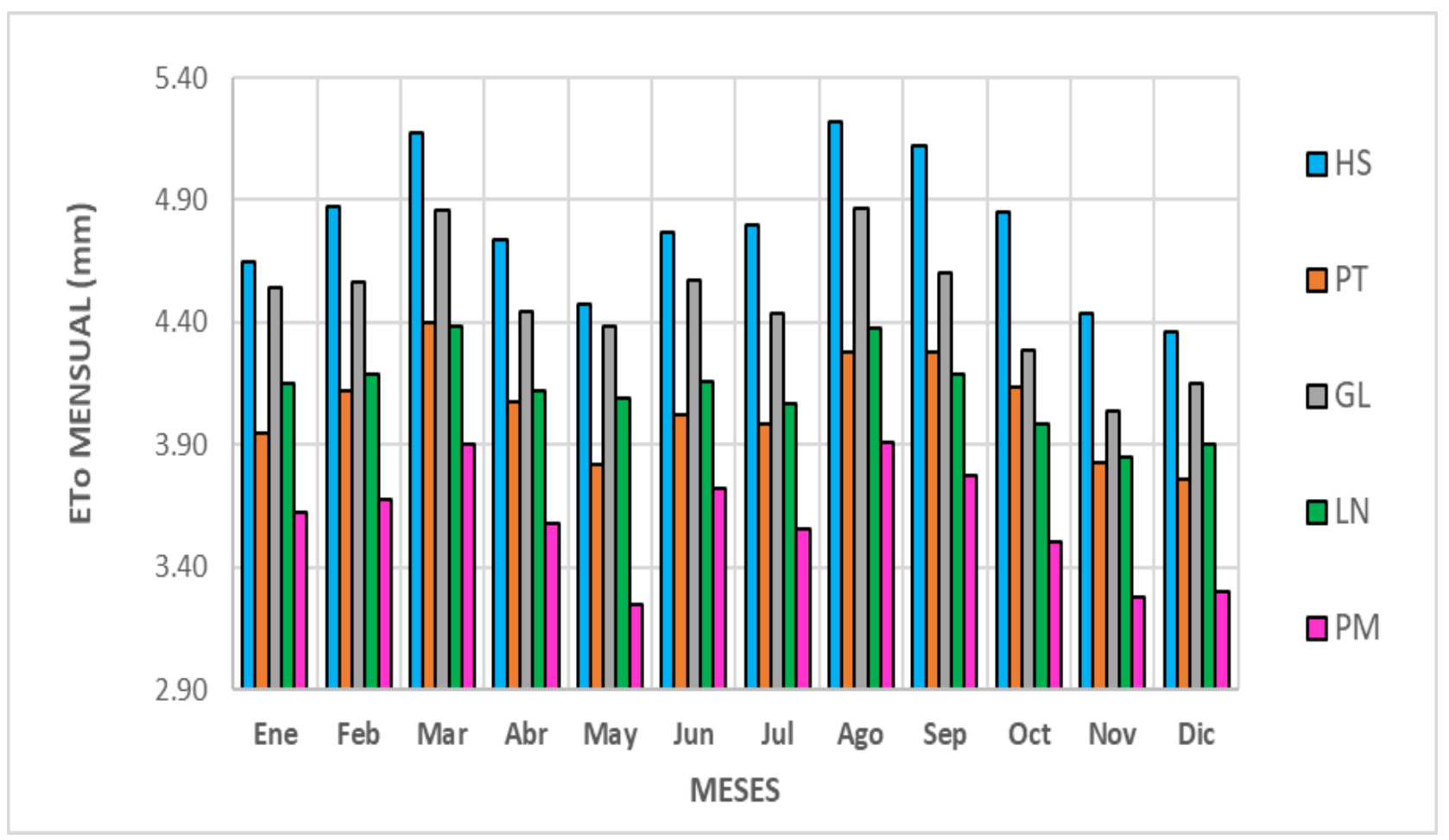

Figura 4. ETo promedio mensual para un año seco en la zona productora de arroz.

Se observó que para las estaciones Ing. Bengala y Ciat Quilichao se obtuvieron estimaciones superiores a 0.5 en el coeficiente de determinación en todos los modelos. De hecho, las estimaciones entre los modelos Penman-Monteith y Priestley-Taylor presentó valores de 0.597 de $R^{2}$ para Ing. Bengala y 0.834 para Ciat Quilichao (Figura 5) y valores inferiores a 0.35 de $R^{2}$ para Ing. Cauca, Independencia e Ing. Miranda. 
Tecnología y

Ciencias $\stackrel{\unlhd}{\unlhd}$ gua
2022, Instituto Mexicano de Tecnología del Agua

Open Access bajo la licencia CCBY-NC-SA 4.0

(https://creativecommons.org/licenses/by-nc-sa/4.0/)

\section{Ciat Quilichao}

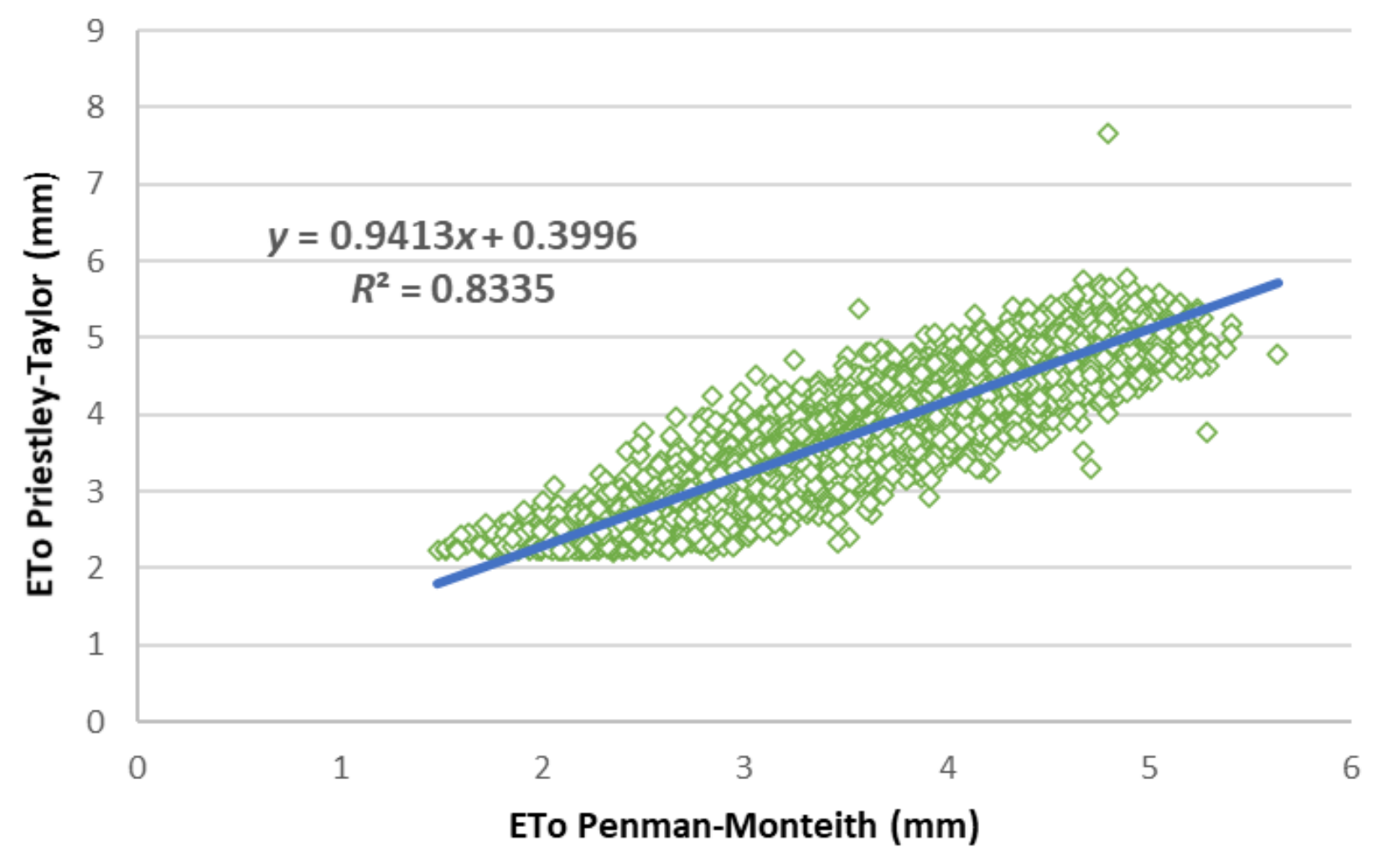

Figura 5. Regresión Lineal Entre PM y PT para la estación Ciat Quilichao.

El modelo HS exhibió coeficientes de determinación bajos de la siguiente forma: valores de $R^{2}$ igual a $0.376,0.290$ y 0.268 para las estaciones Ing. Cauca, Independencia e Ing. Miranda, respectivamente, no así para la estación Ing. Bengala, donde su coeficiente de determinación fue de 0.8264 (Figura 6). Asimismo, el modelo de García y 
Tecnología y

Ciencias ฐึgua
2022, Instituto Mexicano de Tecnología del Agua

Open Access bajo la licencia CC BY-NC-SA 4.0

(https://creativecommons.org/licenses/by-nc-sa/4.0/)

López presentó un rango de valores de $R^{2}$ entre 0.42 y 0.29 para las tres estaciones mencionadas antes.

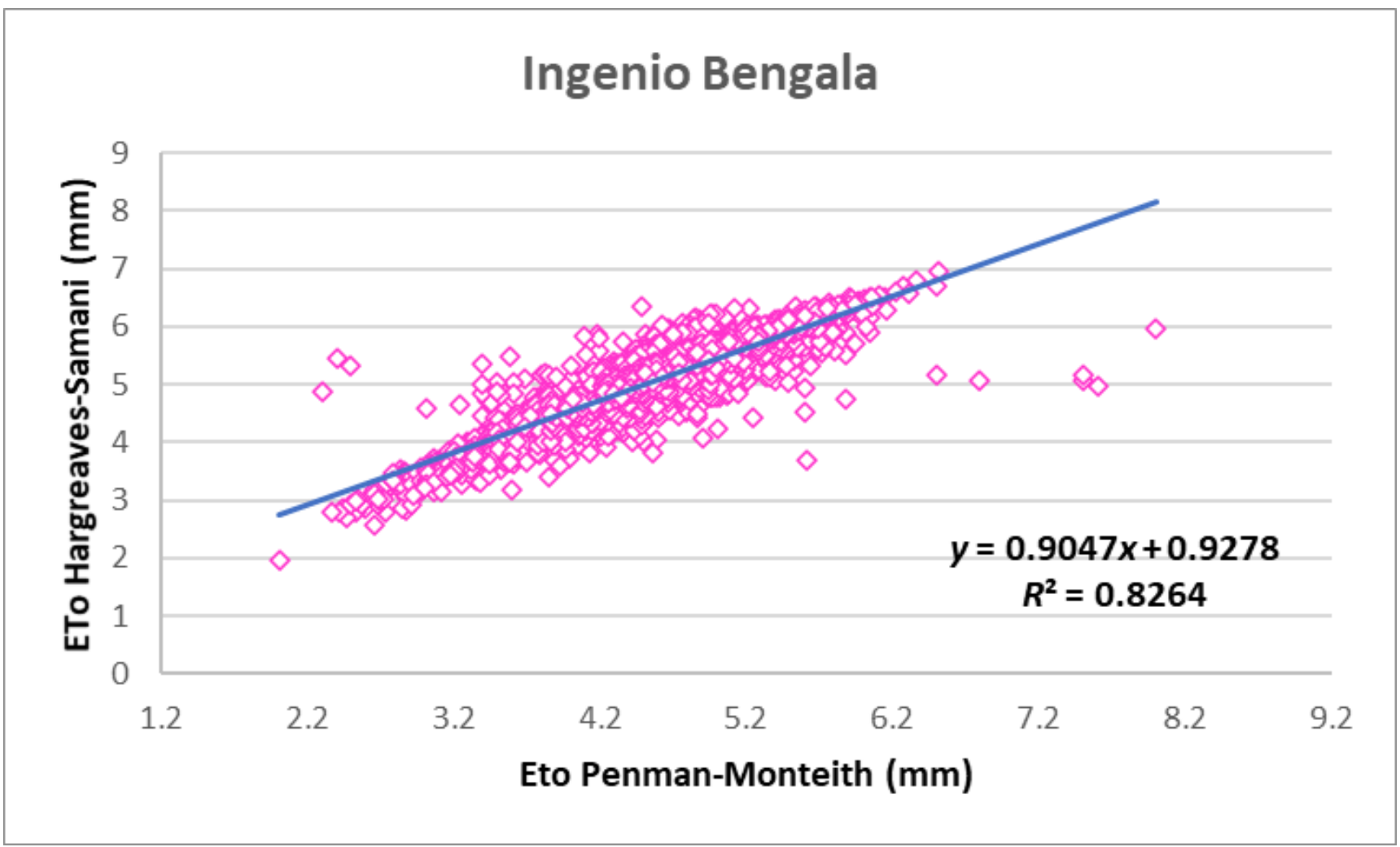

Figura 6. Regresión lineal entre PM y HS para la estación Ing. Bengala.

Linacre se manifestó con los valores más bajos de $R^{2}$ para Independencia e Ing. Cauca (Figura 7), aunque para Ing. Miranda exhibió un valor de 0.330 de $R^{2}$. 
Tecnología y

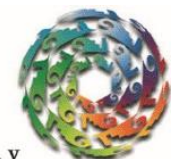

Ciencias $\approx$ Agua
2022, Instituto Mexicano de Tecnología del Agua

Open Access bajo la licencia CC BY-NC-SA 4.0

(https://creativecommons.org/licenses/by-nc-sa/4.0/)

\section{Ingenio Cauca}

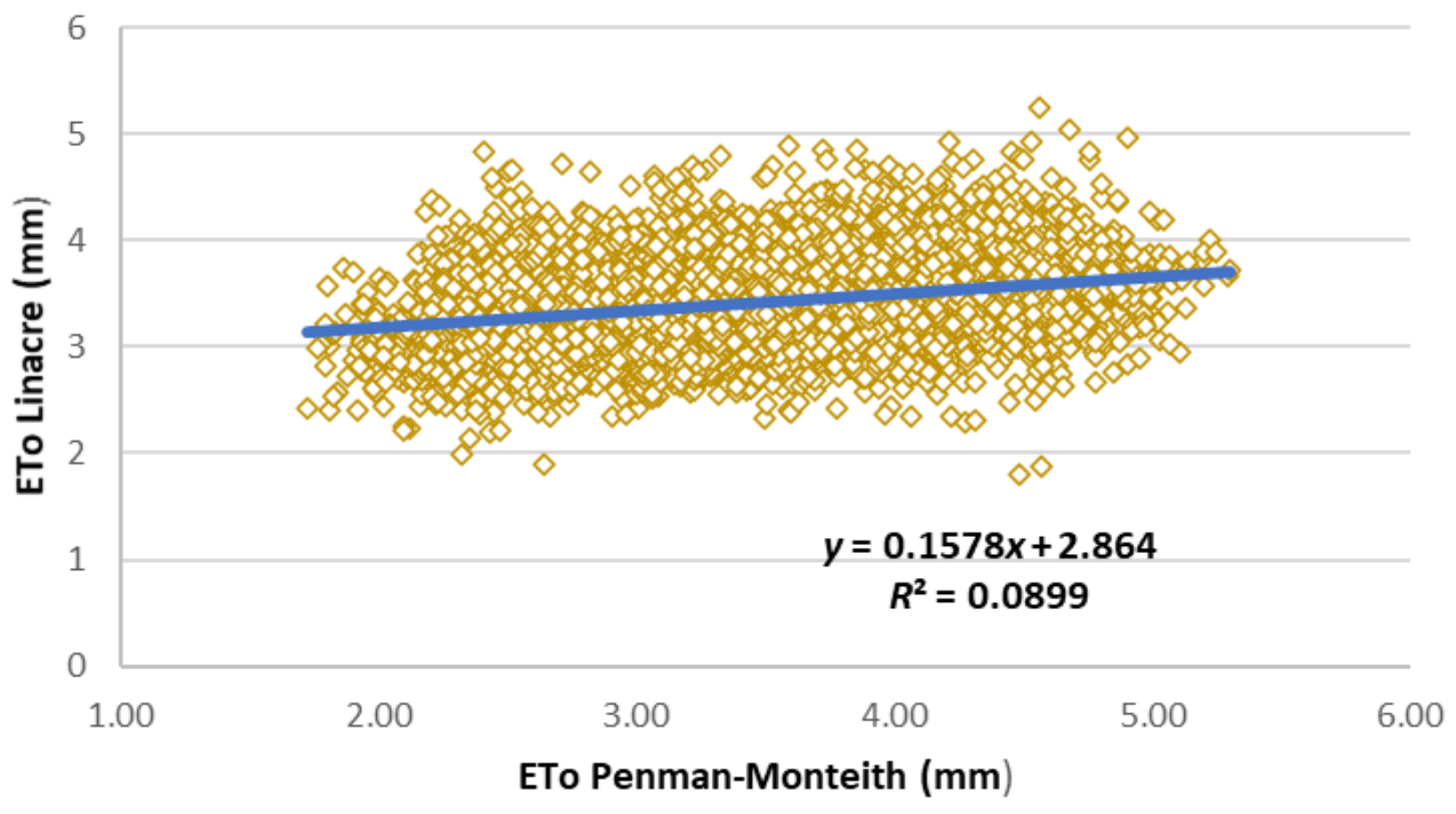

Figura 7. Regresión lineal entre PM y LN para la estación Ing. Cauca.

Los intervalos de confianza en el que se estimaron los coeficientes de las regresiones lineales están basados en un $95 \%$ de confianza (Figura 5 , Figura 6 y Figura 7, Tabla 2), y se observa que los valores del intercepto "a" y "b" están dentro de los límites permitidos para dicho nivel de confianza.

Tabla 2. Intervalo de confianza al $95 \%$. 
Tecnología y

Ciencias $\approx$ Agua
2022, Instituto Mexicano de Tecnología del Agua

Open Access bajo la licencia CC BY-NC-SA 4.0

(https://creativecommons.org/licenses/by-nc-sa/4.0/)

\begin{tabular}{|c|c|c|c|c|c|c|c|}
\hline \multirow[b]{2}{*}{ Modelos } & \multirow[b]{2}{*}{ Estación } & \multirow[b]{2}{*}{$\begin{array}{c}\text { intercepto } \\
a\end{array}$} & \multirow{2}{*}{$\begin{array}{c}\text { Pendiente } \\
\text { b }\end{array}$} & \multicolumn{2}{|c|}{$\begin{array}{l}\text { Intervalo de } \\
\text { confianza a }\end{array}$} & \multicolumn{2}{|c|}{$\begin{array}{l}\text { Intervalo de } \\
\text { confianza b }\end{array}$} \\
\hline & & & & $\begin{array}{c}\text { Límite } \\
\text { inferior } \\
a\end{array}$ & $\begin{array}{c}\text { Límite } \\
\text { superior } \\
\text { a }\end{array}$ & $\begin{array}{c}\text { Límite } \\
\text { Inferior } \\
\text { b }\end{array}$ & $\begin{array}{c}\text { Límite } \\
\text { superior } \\
\text { b }\end{array}$ \\
\hline PT-PM & $\begin{array}{c}\text { Ciat } \\
\text { Quilichao }\end{array}$ & 0.3996 & $0.9413 x$ & 0.371 & 0.429 & 0.933 & 0.949 \\
\hline HS-PM & $\begin{array}{c}\text { Ing. } \\
\text { Bengala }\end{array}$ & 0.9278 & $0.9047 x$ & 0.892 & 0.964 & 0.897 & 0.913 \\
\hline LN-PM & $\begin{array}{c}\text { Ing. } \\
\text { Cauca }\end{array}$ & 2.8640 & $0.1578 x$ & 2.829 & 2.899 & 0.148 & 0.168 \\
\hline \multicolumn{8}{|c|}{ HS: ecuación de Hargreaves-Samani } \\
\hline \multicolumn{8}{|c|}{ PT: ecuación de Priestley-Taylor } \\
\hline \multicolumn{8}{|c|}{ LN: ecuación Linacre } \\
\hline PM: Pe & nont & & & & & & \\
\hline
\end{tabular}

Para este estudio, el error promedio del modelo HS fue de $1.38 \mathrm{~mm}$ día$^{-1}$, lo cual se exhibe como aceptable; aunque se evidencia como el error promedio más alto de todos los modelos, respecto a PM (Tabla 3). A su vez, este índice permite presentar que el modelo de Linacre puede ser un sustituto del método PT, pues su RMSE promedio de las cinco estaciones para el área de estudio fue $0.71 \mathrm{~mm} \mathrm{día-1}^{-1}$ (Tabla 3). 
Tabla 3. Raíz cuadrada del cuadrado medio del error (RMSE) de la EToPM respecto a HS, PT, GL y LN para cada estación

\begin{tabular}{|c|c|c|c|c|c|}
\hline Ecu & $\begin{array}{c}\text { PM-Ing. } \\
\text { Bengala }\end{array}$ & $\begin{array}{c}\text { PM-Ing. } \\
\text { Cauca }\end{array}$ & $\begin{array}{c}\text { PM-Ciat } \\
\text { Quilichao }\end{array}$ & $\begin{array}{c}\text { PM- } \\
\text { Independencia }\end{array}$ & $\begin{array}{c}\text { PM-Ing. } \\
\text { Miranda }\end{array}$ \\
\hline HS & 0.55 & 1.39 & 1.30 & 2.01 & 1.66 \\
\hline PT & 0.35 & 0.59 & 0.36 & 1.13 & 0.76 \\
\hline GL & 0.64 & 0.66 & 0.71 & 2.69 & 0.73 \\
\hline LN & 1.03 & 0.59 & 0.60 & 0.75 & 0.60 \\
\hline HS: ecuación de Hargreaves-Samani \\
\hline PT: ecuación de Priestley-Taylor \\
\hline \\
\hline
\end{tabular}

En cuanto a la eficiencia de Nash-Sutcliffe (NSE), se halló poca precisión en la estimación de la ETo en todos los modelos por estaciones. No obstante, se identifican dos resultados positivos significativos: PM-PT para Ciat Quilichao y PM-LN para Ing. Bengala (Tabla 4); no hay duda de que el modelo PT en la estación Ciat Quilichao es el único modelo que obtuvo un valor de eficiencia positivamente alto y tuvo menor error (Tabla 3 y Tabla 4). 
Tabla 4. Eficiencia de Nash-Sutcliffe (NSE) de la ETo-PM respecto a HS, PT, GL y LN para cada estación

\begin{tabular}{|c|c|c|c|c|c|}
\hline Ecu & $\begin{array}{l}\text { PM-Ing. } \\
\text { Bengala }\end{array}$ & $\begin{array}{l}\text { PM-Ing. } \\
\text { Cauca }\end{array}$ & $\begin{array}{l}\text { PM-Ciat } \\
\text { Quilichao }\end{array}$ & $\begin{array}{c}\text { PM- } \\
\text { Independencia }\end{array}$ & $\begin{array}{l}\text { PM-Ing. } \\
\text { Miranda }\end{array}$ \\
\hline HS & -0.09 & -3.60 & -2.38 & -8.21 & -5.84 \\
\hline PT & -0.70 & -2.06 & 0.74 & -3.84 & -2.97 \\
\hline GL & -0.46 & -0.03 & -0.02 & -15.52 & -0.34 \\
\hline LN & 0.42 & 0.02 & -0.88 & -0.76 & -2.07 \\
\hline \multicolumn{6}{|c|}{ HS: ecuación de Hargreaves-Samani } \\
\hline \multicolumn{6}{|c|}{ PT: ecuación de Priestley-Taylor } \\
\hline \multicolumn{6}{|c|}{ GL: ecuación de García-López } \\
\hline \multicolumn{6}{|c|}{ LN: ecuación Linacre } \\
\hline
\end{tabular}

\section{Diagrama de cajas}

Los resultados obtenidos de los análisis anteriores lograron identificarque el modelo Priestley-Taylor es uno de los métodos que tiene alta afinidad con el método estándar; pero otra característica relevante de la discusión es la distribución del conjunto de datos de cada modelo por estación (Figura 8). 


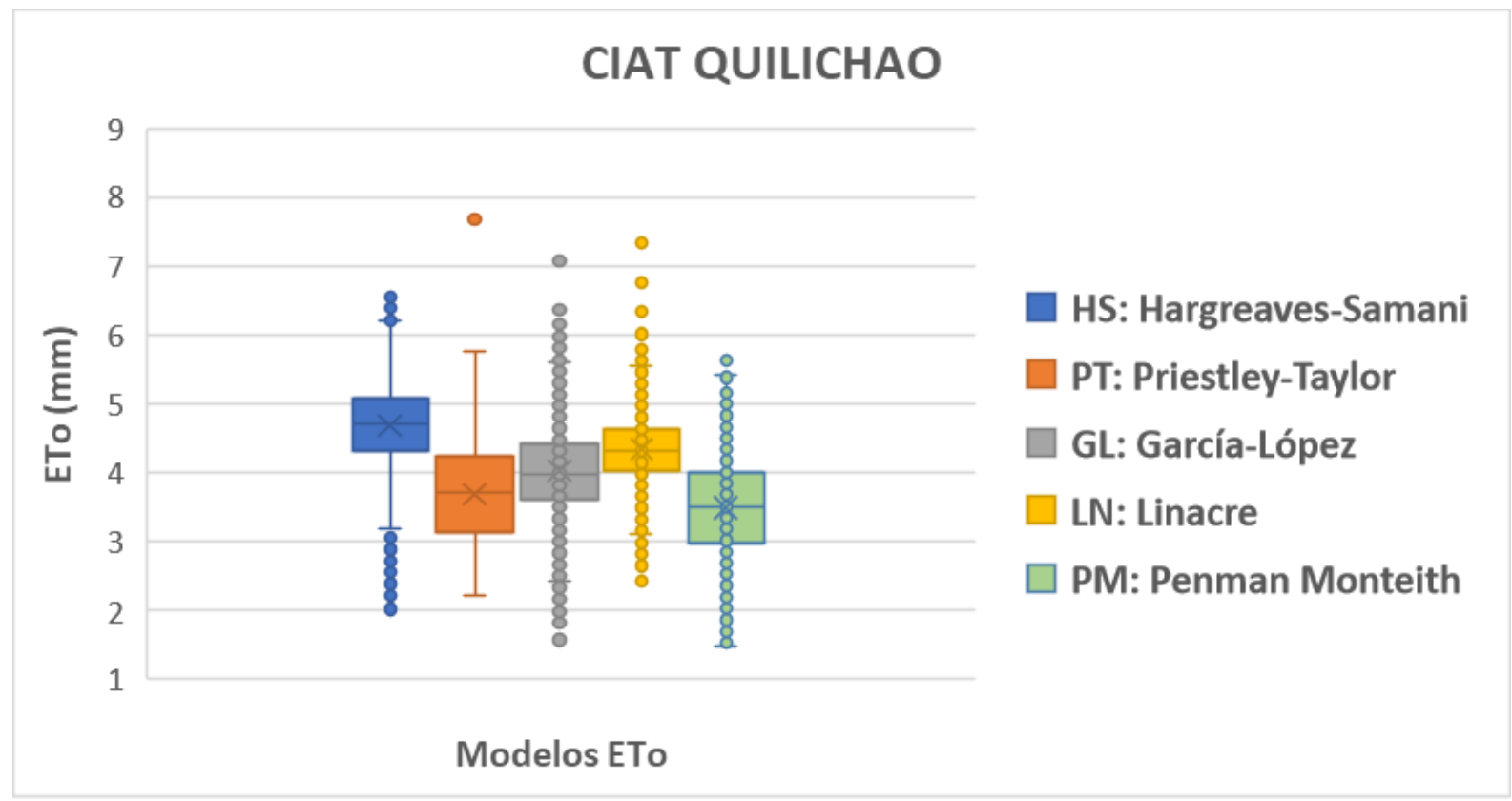

Figura 8. Estimaciones de la ETo para la estación Ciat Quilichao.

Existe una gran variabilidad en las estimaciones de la ETo diaria, por lo que es notorio la posición desigual de las cajas entre sí, y tras las estimaciones de la NSE se identificó que para la estación independencia no se describe cuantitativamente la precisión de los modelos usados contra el modelo estándar (Figura 9). 


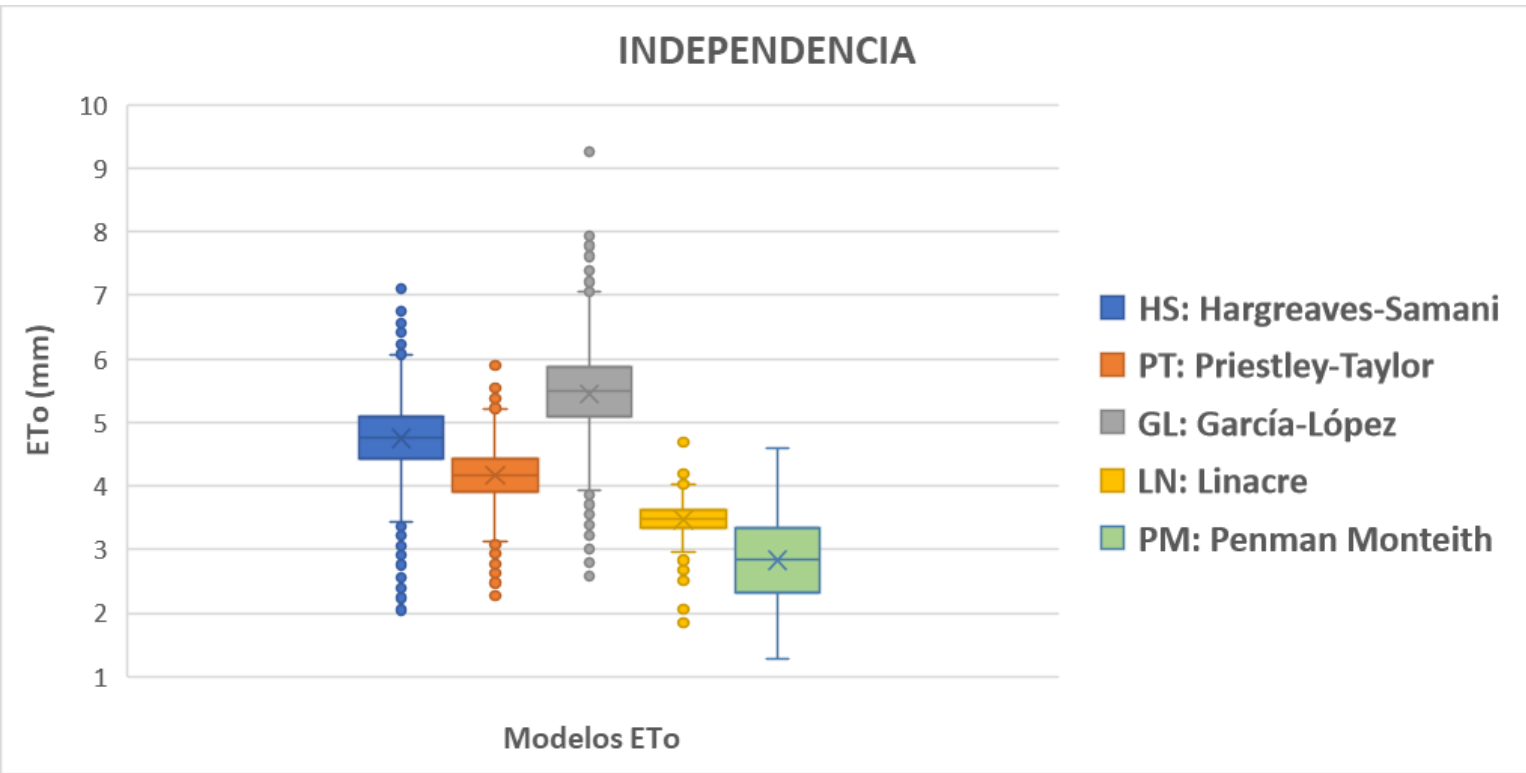

Figura 9. Estimaciones de la ETo para la estación Independencia.

De la estación Ing. Miranda, los modelos LN y PT tienen una varianza de 0.12 y un comportamiento similar en su desviación estándar de 0.34 y $0.35 \mathrm{~mm}$ día $^{-1}$, con un conjunto de datos que coinciden con las estimaciones hechas por el método estándar (Figura 10). De hecho, el modelo LN-PM presentó mejores resultados que los demás modelos para esta estación (Tabla 3). 


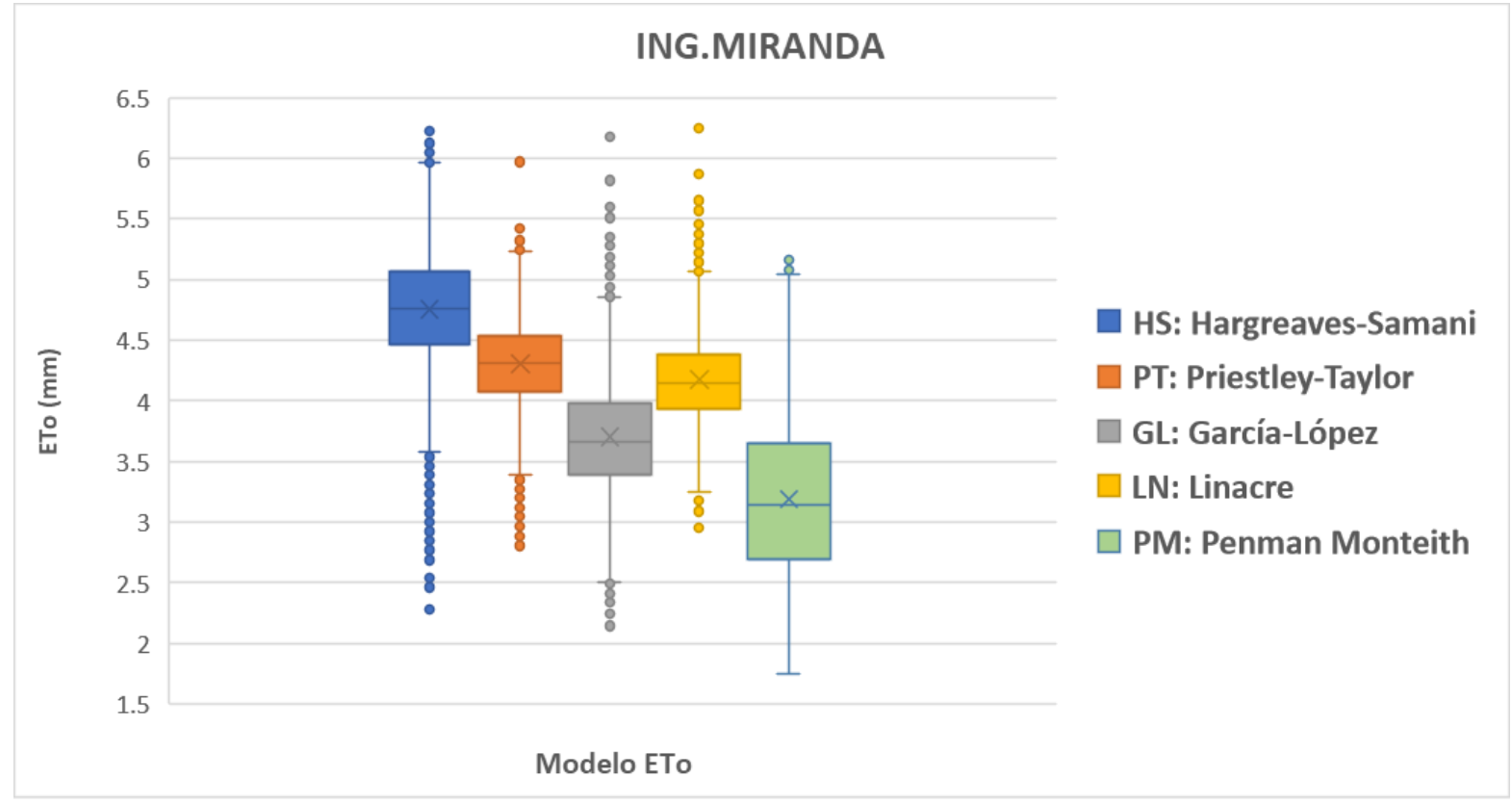

Figura 10. Estimaciones de la ETo para la estación Ing. Miranda.

La estación Ing. Bengala exhibe que las estimaciones hechas por el modelo PM y HS tienen una varianza de 0.281 y 0.278 , respectivamente, y una desviación estándar similar de $0.53 \mathrm{~mm}$ día $^{-1}$ (Figura 11). Sin embargo, para este caso fue el mismo método estándar el que presentó más observaciones que se alejan de manera poco usual del resto de los datos. En cambio, los modelos PT, GL y LN en su conjunto de datos están más cercanos a la media; a su vez, obtuvieron los errores más bajos para esta estación con el índice RMSE. 


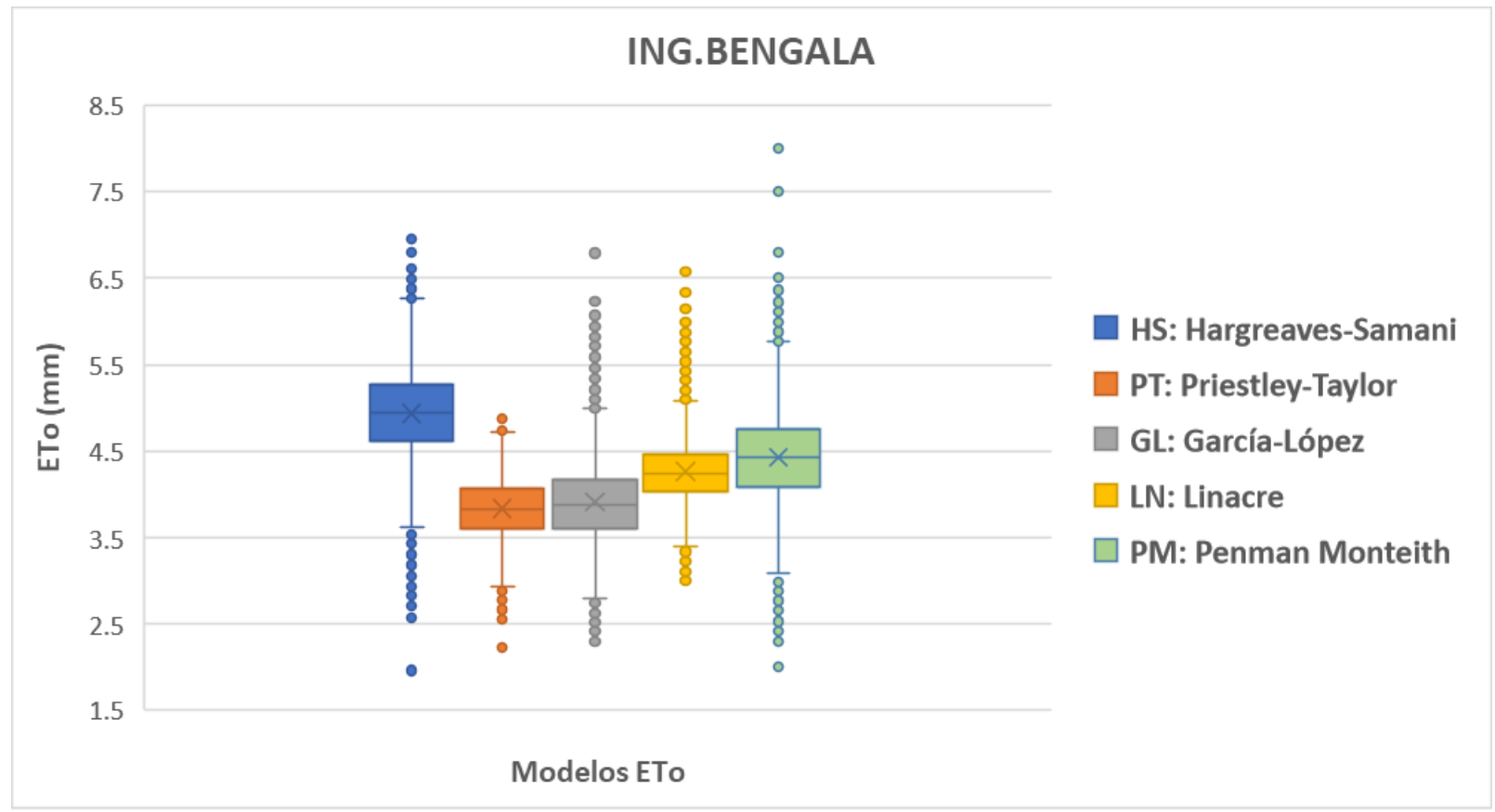

Figura 11. Estimaciones de la ETo para la estación Bengala.

Los valores obtenidos de la raíz cuadrada del cuadrado medio del error para la estación Ing. Cauca fueron inferiores a $1 \mathrm{~mm}$ para los modelos PT, GL y LN (Figura 12, Tabla 3), los cuales concuerdan con el $95 \%$ de las estimaciones realizadas con el modelo GL con el modelo estándarpara la estación Ing. Cauca. En cambio, el 75 \% de los resultados obtenidos del modelo de Linacre coindicen con el $50 \%$ de los valores obtenidos del modelo PM. Del mismo modo, Hargreaves-Samani posee la media más alta con valores atípicos y extremos, que comparando éste con el modelo estándar se podría indicar que HS no es un método conveniente para dicha estación, ya que PM los considera dentro de su varianza. 


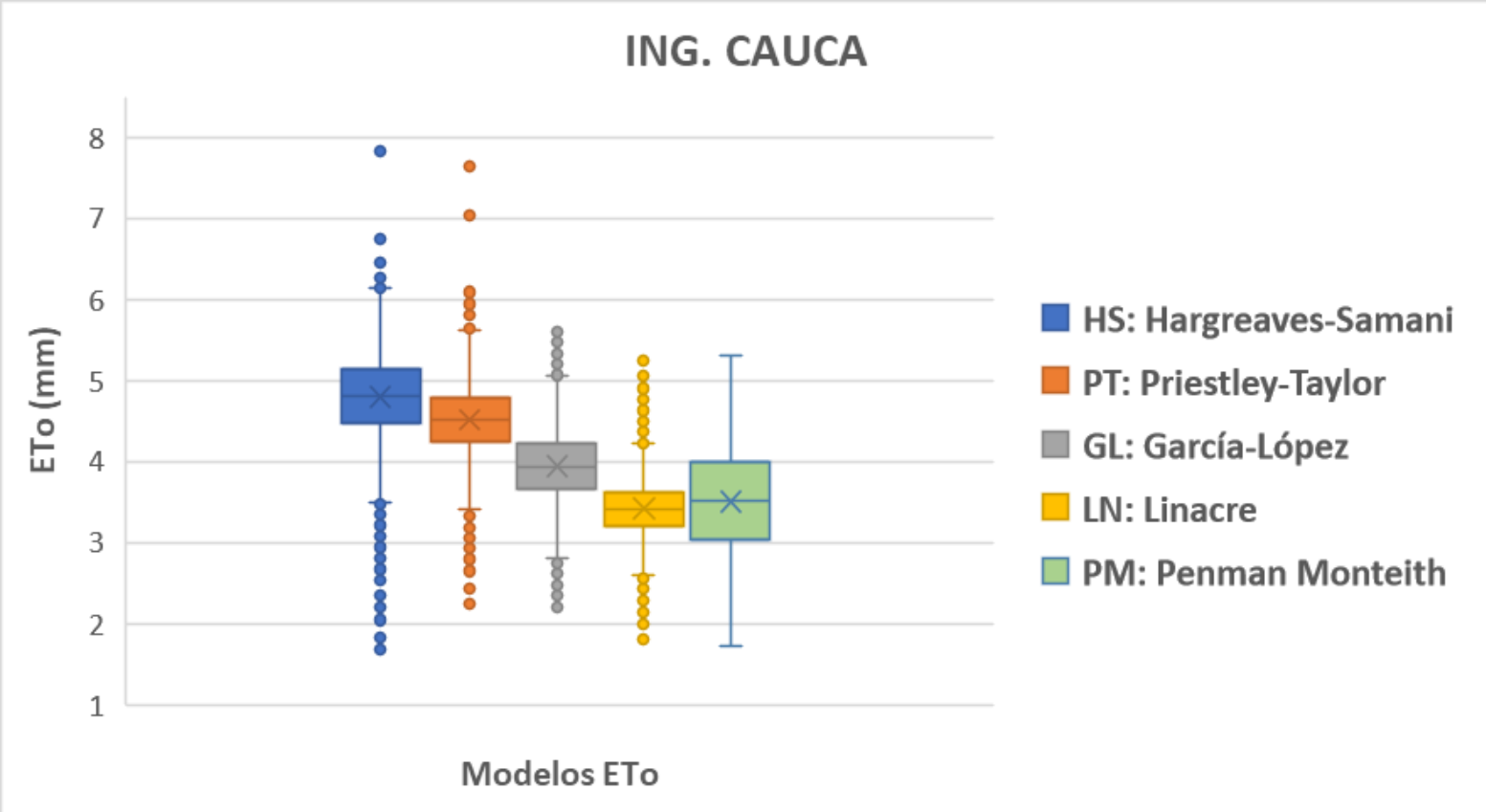

Figura 12. Estimaciones de la ETo para la estación Ing. Cauca.

Los gráficos exhibidos anteriormente permiten identificar que los modelos más cercanos al método estándar para las cinco estaciones fueron el PT y LN, pues presentaron estadísticas idénticas, mejores ajustes o menores errores.

\section{Discusión}


El modelo PM a nivel general se manifestó en un intervalo de 3 a $3.8 \mathrm{~mm}$ mes $^{-1}$ y HS se exhibió como el método que presenta promedios mensuales superiores en todos los años elegidos. En el año lluvioso, junio y diciembre se presenta como los meses con los promedios más bajos en todos los métodos; esto acontece por el fenómeno de la zona de convergencia intertropical, que influye en el comportamiento bimodal de la precipitación para el país (Figura 2).

Marzo y agosto del año seco presentan los promedios más altos y entre métodos los valores tienden a ser similares (Figura 4); de acuerdo con el registro del IDEAM, agosto es el mes que mayor evaporación registra en los datos históricos.

Un estudio hecho por Gunston y Batchelor (1983) para comparar los modelos PM y PT estimó la evapotranspiración de referencia a nivel mensual en los países tropicales, y registró un desempeño aceptable de $R^{2}=0.87$ para 126 meses húmedos; $R^{2}=0.90$ para 86 meses de lluvias intermedias, y $R^{2}=0.48$ en 148 meses de sequía. Otro ejemplo fue ejecutado por Qiu et al. (2019), en la ciudad Nanjing, de la provincia Jiangsu en China, cuyo modelo PT estimó razonablemente la ETo para la zona de producción de arroz con unos coeficientes de determinación de 0.92 y 0.96 para dos estaciones. Este modelo ha sido exitoso, después del método PM, gracias a la radiación neta $(R n)$, que es una variable meteorológica que afecta a la ET-referencia a corto plazo en el sistema 
de producción de arroz. No obstante, cuando una estación meteorológica tiene un conjunto de datos limitados de temperatura y radiación solar, la mejor opción para ese caso parece ser el uso de PT, que es una simplificación del método original Penman-Monteith (Senthelas, Gillespie, \& Santos, 2010).

Respecto al modelo HS, Awal, Habibi, Fares y Deb (2020) indican que la ETo diaria estimada con el modelo Hargreaves-Samani en diferentes estaciones del oeste de Texas, EUA, reveló más del 0.7 de coeficiente de determinación entre este modelo respecto al de PM; es decir, que existe un alto nivel de concordancia entre ambos métodos para esta zona.

En cambio, los resultados del modelo $G L$, a pesar de ser bajos son considerados aceptables, pues este modelo ha sido empleado en otros estudios a nivel mensual y anual (Sánchez, 2000); es una opción para cuando no se pueden emplear otros métodos debido a la carencia de registros de brillo solar.

En este sentido, los resultados del modelo LN se corroboran con los encontrados por Issaka et al. (2017), quienes indicaron que este modelo se posicionó en último lugar con un rendimiento moderado de $R^{2}=0.47$ entre la evaluación de seis modelos respecto al estándar, cuyos datos diarios utilizados fueron los de la estación meteorológica del Aeropuerto Internacional de Doha, Qatar, en un período de 30 años. Otro ejemplo es el de Araújo, Oliveira, Araújo, Ledo y Silva (2010), quienes evaluaron el desempeño de los métodos de estimación de la evapotranspiración de referencia en las ciudades del estado de Ceará, encontrando un 
desempeño aceptable para este método de $R^{2}=0.934$. El rendimiento de este modelo se debe a que utiliza sólo datos de temperatura del aire y altitud como la variable del sitio, limitando la representatividad de las condiciones climáticas para propósitos de estimación de la ETo (Pereira, Yanagi, Mello, Silva, \& Silva, 2009).

De los 20 modelos lineales desarrollados, se identificó que el valor de la pendiente es estadísticamente diferente de cero, pero no mayor a uno, y por lo tanto la regresión es significativa. De hecho, el valor " $p$ " fue similar $(<2 \mathrm{e}-16)$ para todas las regresiones; es un valor inferior al valor de "p" crítico (0.05).

De los valores obtenidos del índice RMSE entre los modelos PM-HS se identifican similitudes expresadas por Amatya, Skaggs y Gregory (1995), quienes obtuvieron un intervalo de errores de RMSE de 0.87 a $1.37 \mathrm{~mm}$ día $^{-1}$; en cambio, el error promedio de RMSE entre el modelo HS y PM para 12 estaciones en Khuzestan, suroeste de Irán, fue de $1.17 \mathrm{~mm}$ día $^{-1}$ (Rahimi, 2008). Sin embargo, Lavado, Lhomme, Labat, Loup-Guyot y Boulet (2015) presentan $0.50 \mathrm{~mm}$ día $^{-1}$ como error de la media del modelo HS respecto al modelo de referencia PM para ocho estaciones en Perú. Si bien es notorio, la estación Independencia exhibió los resultados más altos en este índice en los modelos HS, PT y GL contra PM; esto permite enunciar que tales métodos se pueden recomendar para el cálculo de la ETo en otro tipo de escala para dicha estación (Martínez, Boueri, \& Escalona, 2005).

Los resultados de LN son valores similares a los reportados por Lavado et al. (2015), quienes exhiben valores de 0.42 a $1.05 \mathrm{~mm}$ día-1 
para ocho estaciones en la cuenca amazónica andina. Esto ha sido posible gracias a que se requiere sólo la temperatura media $(T)$ y el punto de rocío $(\mathrm{Td})$, y es un modelo útil en una amplia gama de climas expuesto por Linacre (1977); no es necesario medir el punto de rocío si se dispone de temperaturas extremas diarias, pues éstas permiten hacer una estimación aproximada.

Para el alcance de este trabajo, el valor obtenido de la NSE para Ciat Quilichao resulta ser mucho mayor que el reportado por Celestin, Qi, $\mathrm{Li}$, Yu y Cheng (2020), quienes evaluaron 32 ecuaciones de evapotranspiración de referencia contra el método Penman-Monteith en el corredor Hexi, en el noroeste de China; también concluyen que el modelo HS resulta ser un buen sustituto de PM a escala mensual y para dicho lugar. Por otro lado, del modelo Linacre no se halló trabajos que indaguen sobre la bondad del modelo.

\section{Diagrama de cajas}

El modelo PM exhibe un rango de 3 a $4 \mathrm{~mm} \mathrm{día}^{-1}$ de la ETo estimada para la estación Ciat Quilichao, la cual coinciden con el 50 \% de los resultados obtenidos por el modelo PT. Ambos modelos exhiben correspondencia similar para esta estación, ya que los respectivos valores de varianza son de $0.50 \mathrm{~mm}^{2}$ para PM y $0.53 \mathrm{~mm}^{2}$ para PT, y la desviación estándar es 
de $0.71 \mathrm{~mm}$ día-1 para PM y $0.73 \mathrm{~mm}^{-1}$ día $^{-1}$ para $\mathrm{PT}$, lo que permite inferir que en ausencia de datos para estimar este parámetro con el modelo de PM se puede emplear el PT, tal cual se corroboró con el índice de la eficiencia de Nash-Sutcliffe (Tabla 4 y Figura 8 ), similitud que también divulgan Lu, Sun, McNulty y Amatya (2005) al comparar seis modelos diarios para calcular la ETo respecto al modelo PM en el sureste de EUA.

De hecho, Linacre se puede considerar como un tercer modelo para estimar la ETo en la estación Independencia, ya que ha manifestado un intervalo de resultados similares al del PM. En cambio, de los otros modelos se puede mencionar que no coinciden con PM, pues la varianza para GL, PT y HS es de $0.60,0,40$ y $0.53 \mathrm{~mm}^{2}$, respectivamente (Figura 9). De modo comparativo, Vicente-Serrano et al. (2014) exhiben que para la época de otoño, el modelo Linacre mostró valores de ETo igual y más altos que el método PM, pero se observaron valores inferiores estimados de LN para la sección de verano respecto al método estándar. Esto es posible, ya que cada ecuación fue creada para unas condiciones climáticas específicas que pueden diferir de las presentadas por cada estación; así, el comportamiento de cada modelo es muy cambiante dependiendo del lugar y época del año en la que se utilice (Sánchez, 2000).

No obstante, en la estación Ing. Miranda, el modelo PM presenta un conjunto de datos entre 1.7 y $5.1 \mathrm{~mm}^{2} \mathrm{~d}^{-1}$, con pocos valores extremos entre 5.2 y $5.5 \mathrm{~mm}$ día$^{-1}$; eso indica que el uso de este modelo para esta estación exhibe algunos valores alejados de su media en más de dos unidades (Figura 10). Una investigación similar realizada en Macedonia Occidental, Grecia, por Efthimiou, Alexandris, Karavitis y Mamassis (2013), presentó que los modelos LN y PT reportaron el 79 y $89 \%$ de 
Tecnología y

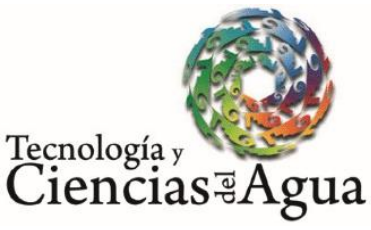

2022, Instituto Mexicano de Tecnología del Agua

Open Access bajo la licencia CC BY-NC-SA 4.0

(https://creativecommons.org/licenses/by-nc-sa/4.0/)

coincidencia en las estimaciones diarias de 34 años de la ETo respecto a las del Penman-Monteith.

Tanto el Modelo PM y Hs sirven para caracterizar dicha variable en la estación Ing. Bengala; esto se confirma por el coeficiente de determinación entre ambos métodos que fue de 0.826 (Figura 6). PM se ve influenciado por varios parámetros que, de acuerdo con los experimentos hechos por Tyagi, Sharma y Luthra (2000), existe una probabilidad del 93.4 \% que un modelo diferente al de PM tenga coincidencia con él (Figura 11); pero Lang et al. (2017) aluden que la ecuación de HS se puede usar más fácilmente que PT para estimar la ETo en el suroeste de China, ya que la primera requiere menos parámetros para estimar la ETo que la segunda.

En la estación Ing. Cauca, los modelos PT y GL se caracterizan por presentar 0.43 y $0.44 \mathrm{~mm}$ día $^{-1}$ de desviación estándar, pero con una media y conjunto de datos diferentes. Gong, Xu, Chen, Halldin y Chen (2006) exponen que las variables climáticas en las mediciones diarias de la ETo entre el modelo PM y GL manifiestan que la humedad relativa es el factor meteorológico más sensible, seguido de la velocidad del viento, la radiación solar y la temperatura. En la Figura 12, el método que es más parecido al de PM es el de LN. El modelo HS presenta la mayor dispersión en la distribución de los datos; casos similares los reportan Pérez y Castellví (2002), quienes enuncian que este modelo exhibe los mayores valores atípicos en el cálculo de la ETo en la región de Cataluña en España.

En síntesis, la relación de las ecuaciones Penman-Monteith y Priestley-Taylor expone su validez cuando se disponen de registros 
necesarios para su aplicación a nivel diario. Dos anotaciones importantes las expresan Vicente-Serrano et al. (2014), quienes indican que los modelos basados en las temperaturas como Hargreaves-Samani suelen subestimar la ETo para varias estaciones de un solo lugar y que algunos métodos pueden mostrar la mejor concordancia con el modelo PM, pero podría tener la menor concordancia en una estación diferente. Sin embargo, es importante manifestar que los modelos restantes constituyen una herramienta admisible cuando no se cuenta con los datos climatológicos suficientes o de procedencia confiable, tal y como lo señalan Allen et al. (1998), quienes señalan que se pueden sugerir diversos métodos para el cálculo de la ETo con base en el tipo de información climática que se tenga.

\section{Conclusiones}

El modelo Penman-Monteith de la FAO estima la evapotranspiración de referencia diaria (ETo) al considerar un conjunto completo de datos meteorológicos y, a su vez, es la principal restricción para su uso en lugares donde faltan datos; situación que se da en el sur del departamento del Valle del Cauca, y con pocas estaciones meteorológicas, 
donde es frecuente la falta de datos sobre velocidad del viento, la humedad relativa y la radiación solar. No existe ningún estudio previo que evalúe el rendimiento de los diversos métodos para estimar la evapotranspiración de referencia diaria en esta región de Colombia.

El modelo Priestley-Taylores un buen método entre los estudiados; manifestó una alta similitud con los valores obtenidos respecto al modelo estándar Penman-Monteith con el índice de la raíz cuadrada del cuadrado medio del error (RMSE) para todas las estaciones. Con el índice de la Eficiencia de Nash-Sutcliffe sólo presentó un valor altamente aceptable para la estación Ciat Quilichao. Otro modelo útil para estimar la evapotranspiración de referencia diaria es el de Linacre; al igual que Priestley-Taylor, ambos métodos son ideales, ya que son modificaciones del modelo estándar.

Existe evidencia de que el uso a nivel diario del modelo García y López es limitado, a pesar de ser una ecuación adaptada para el trópico, cuyos factores climatológicos son de fácil adquisición. Respecto al modelo Hargreaves-Samani, se presenta como un método aceptable para la estación Ing. Bengala; sin embargo, este modelo junto al de García y López no son ideales para la estación independencia, ya que los resultados obtenidos por el método RMSE Y NSE no son acordes con lo esperado en los alcances de este trabajo.

\section{Agradecimientos}

Los autores agradecen al Consejo Nacional de Ciencia y Tecnología (Conacyt) y a la Dirección General de Estudios de Posgrado de la 
Universidad Autónoma Chapingo por proporcionar apoyo para la elaboración de la presente investigación, que se ejecutó durante el desarrollo del programa de estudios de la Maestría en Ingeniería Agrícola y Uso Integral del Agua de la primera autora.

\section{Referencias}

Allen, R. G., Pereira, L. S., Raes, D., \& Smith, M. (1998). Crop evapotranspiration. Serie de Riego y Drenaje. FAO-56. Roma, Italia: Organización de las Naciones Unidas para la Alimentación y la Agricultura.

Allen, R. G., Pereira, L. S., Raes, D., \& Smith, M. (2006). Evapotranspiración del cultivo: guías para la determinación de los requerimientos de agua de los cultivos. FAO-56. Roma, Italia: Organización de las Naciones Unidas para la Alimentación y la Agricultura.

Amatya, D. M., Skaggs, R. W., \& Gregory, J. D. (1995). Comparison of methods for estimating Ref-Et. Journal of Irrigation and Drainage Engineering, 121(6), 427-435.

Araújo, E. M., Oliveira, J. B., Araújo, E. M., Ledo, E. R., \& Silva, M. G. (2010). Desempenho de métodos de estimativa de ETo correlacionados com a equação padrão Penman Monteith FAO56, em cidades do estado do Ceará. Revista Científica, 5(2). Araújo, E. M., Oliveira, J. B., Araújo, E. M., Ledo, E. R., \& Silva, M. G. (2010). Desempenho de métodos de estimativa de ETo correlacionados com 
a equação padrão Penman Monteith FAO56, em cidades do estado do Ceará. Revista Acta Tecnológica, 5(2), 84-101.

Awal, R., Habibi, H., Fares, A., \& Deb, S. (2020). Estimating reference crop evapotranspiration under limited climate data in West Texas. Journal of Hydrology: Regional Studies, 28, 1-19. DOI: 10.1016/j.ejrh.2020.100677

Back, A. J. (2008). Desempenho de métodos empíricos baseados na temperatura do ar para a estimativa da evapotranspiração de referência em Urussanga, SC. Irriga, Botucatu, 13(4), 449-466.

Barco, J., Cuartas, A., Mesa, O., Poveda, G., Vélez, J. I., Mantilla, R., Hoyos, C., Mejía, J. F., Botero, B., \& Montoya, M. (2000). Estimación de la evaporación en Colombia. Avances en Recursos Hidráulicos, 7, 43-51.

Caí, J., Liu, Y., Lei, T., \& Santos, P. L. (2007). Estimating reference evapotranspiration with the FAO Penman-Monteith equation using daily weather forecast messages. Agricultural and Forest Meteorology, 145, 22-35.

Celestin, S., Qi, F., Li, R., Yu, T., \& Cheng, W. (2020). Evaluation of 32 simple equations against the Penman-Monteith Method to estimate the reference evapotranspiration in the Hexi Corridor, Northwest China. Water, 12(10), 2772. Recuperado de https://doi.org/10.3390/w12102772

Carvalho, L., Rios, G. F., Miranda, W., \& Castro, N. P. (2011). Evapotranspiração de referência: uma abordagem atual de diferentes métodos de estimativa. Pesquisa agropecuária tropical. Agricultural 
Tecnología y

Ciencias Agua
2022, Instituto Mexicano de Tecnología del Agua

Open Access bajo la licencia CC BY-NC-SA 4.0

(https://creativecommons.org/licenses/by-nc-sa/4.0/)

Research In The Tropics, 41(3), 456-465. DOI: 10.5216/Pat.V41i3.12760

Cervantes, O. R., Arteaga, R. R., Vázquez, P. M. A., Ojeda, B. W., \& Quevedo, N. A. (2013). Modelos Hargreaves Priestley-Taylor redes neuronales artificiales en la estimación de la evapotranspiración de referencia. Ingeniería, Investigación y Tecnología, 14(2), 163-176.

Dane y Fedearroz. (2017). 40 Censo Nacional Arrocero 2016. Boletín técnico. Bogotá, Colombia: Dane y Fedearroz.

De-Carvalho, A., De-Carvalho, M. L., Vianello, R., Sediyama, G., DeOliveira, M. A., \& De-Junior, S. (2013). Geostatistical improvements of evapotranspiration spatial information using satellite land surface and weather stations data. Theoretical and Applied Climatology, 13(12), 155-174.

Djaman, K., Sall, M., Sow, A., Manneh, B. , \& Irmak, S. (2019). Impact of air temperature and relative humidity measured over rice and grass canopies on Penman-Monteith reference evapotranspiration estimates. Journal of Irrigation and Drainage Engineering, 145(1), 06018008. DOI: 10.1061/(ASCE)IR.1943-4774.0001362

Efthimiou, Alexandris, Karavitis, \& Mamassis. (2013). Comparative analysis of reference evapotranspiration estimation between various methods and the FAO56 Penman-Monteith procedure. European Water, 42, 19-34. Efthimiou, N., Alexandris, S., Karavitis, C., \& Mamassis, N. (2013). Comparative analysis of reference evapotranspiration estimation between various methods and the FAO56 Penman-Monteith procedure. European Water, 42, 19-34. 
García, J., \& López, J. (1970). Fórmula para el cálculo de la evapotranspiración potencial adaptada al trópico. Agronomía Tropical, 20(5), 335-345.

Gong, L., Xu, C., Chen, D., Halldin, S., \& Chen, Y. D. (2006). Sensibilidad de la evapotranspiración de referencia Penman-Monteith a variables climáticas clave en la cuenca de Changjiang (río Yangtze). Journal of Hydrology, 329(3-4), 620-629. DOI: 10.1016/j.jhydrol.2006.03.027

Gunston, H., \& Batchelor, C. H. (1983). A comparison of the PriestleyTaylor and Penman methods for estimating reference crop evapotranspiration in tropical countries. Agricultural Water Management, 6, 65-77.

Issaka, A. I., Paek, J., Abdella, K., Pollanen, M., Huda, A. K. S., Kaitibie, S., \& Moustafa, A. T. (2017). Análisis y calibración de relaciones empíricas para estimar la evapotranspiración en Qatar: estudio de caso. Revista de Ingeniería de Riego y Drenaje, 143(2),1-7. DOI: 10.1061/(asce)ir.1943-4774.0001106

Jaramillo, A. (2006). Evapotranspiración de referencia en la región andina de Colombia. Cenicafé, 57(4), 288-298.

Lavado, W., Lhomme, J., Labat, D., Loup-Guyot, J., \& Boulet, G. (2015). Estimación de la evapotranspiración de referencia (FAO PenmanMonteith) con limitados datos climáticos en la Cuenca Andina Amazónica Peruana. Revista Peruana Geo-Atmosférica, 4, 34-36.

Lang, D., Zheng, J., Shi, J., Liao, F., Ma, X., Wang, W., Chen, X., \& Zhang, M. A. (2017). Comparative study of potential evapotranspiration 
estimation by eight methods with FAO Penman-Monteith Method in Southwestern China. Water, 9(734), 1-18.

Linacre, E. T. (1977). A simple formula for estimating evapotranspiration rates in various climates, using temperature data along. Agricultural Meteorology, 18, 409-424.

López, M. J. I., Hess, T. M. \& White, S. M. (2009). Estimation of reference evapotranspiration in a mountainous Mediterranean site using the Penman-Monteith equation with limited meteorological data. Pirineos, 164, 7-31.

Lu, J., Sun, G., McNulty, S., \& Amatya, D. A. (2005). Comparison of six potential evapotranspiration methods for regional use in the southeastern United States. Journal of the American Water Resources Association, 41(3), 621-633.

Magana-Hernandez, F., Ba, K., Guerra-Cobian, M. Y., \& Víctor, H. (2013). Estimación del hidrograma de crecientes con modelación determinística y precipitación derivada de radar. Agrociencia, 47(8), 739-752.

Martínez, B. R., \& Mendivelso, L. D. (2004). Aspectos del medio natural. En: IGAC y CVC. Levantamiento de suelos y zonificación de tierras del Departamento del Valle del Cauca. Tomo I. (pp. 1-541). Bogotá D.C., Colombia. Martínez, B. R., \& Mendivelso, L. D. (2004). Aspectos del medio natural. En: IGAC y CVC. Levantamiento de suelos y zonificación de tierras del Departamento del Valle del Cauca. Tomo I. (pp. 1-541). Bogotá, DC, Colombia: IGAC. 
Tecnología y

Ciencias Agua
2022, Instituto Mexicano de Tecnología del Agua

Open Access bajo la licencia CC BY-NC-SA 4.0

(https://creativecommons.org/licenses/by-nc-sa/4.0/)

Martínez, R., Boueri, M. A., \& Escalona, P. (2005). Correlación entre la evaporación en tina y la evapotranspiración de referencia en cinco estaciones climatológicas de Venezuela. Bioagro, 17(2), 79-83.

Martínez, C. A., \& Pérez, C. O. (2006). Evapotranspiración del cultivo del Arroz bajo riego por aspersión. Ingeniería del Agua, 13(1), 59-69.

Pereira, A. R. (2004). The Priestley-Taylor parameter and the decoupling factor for estimating reference evapotranspiration. Agricultural and Forest Meteorology, 125(3-4), 305- 313, DOI: $10.1016 /$ j.agrformet.2004.04.002

Pereira, D. R., Yanagi, S. N. M., Mello, C. R., Silva, A. M., \& Silva, L. A. (2009). Desempenho de métodos de estimativa da evapotranspiração de referência para a região da Serra da Mantiqueira, MG. Ciência Rural, 39(9), 2488-2493.

Pérez, P., \& Castellví, F. (2002). Análisis de la evapotranspiración a escala local y regional en Cataluña. Ingeniería del Agua, 9(1), 59-72.

Poveda, G., Vélez, J. I., Mesa, O. J., Cuartas, A., Barco, J., Mantilla, R. I., Mejía, J. F., Hoyos, C. D., Ramírez J. M., \& Ceballos, L. I. (2007). Linking long-term water balances and statistical scaling to estimate river flows along the drainage network of Colombia. Journal of Hydrologic Engineering, 12(1), 4-13.

Qiu, R., Liu, C., Cui, N., Wu, Y., Wang, Z., \& Li, G. (2019). Evapotranspiration estimation using a modified Priestley-Taylor model in a rice-wheat rotation system. Agricultural Water Management, 224(105755), 1-13. 
Rahimi, K. A. (2008). Comparative study of Hargreaves's and artificial neural network's methodologies in estimating reference evapotranspiration in a semiarid environment. Irrigation Science, 26, 253-259. DOI: $10.1007 / \mathrm{s} 00271-007-0090-z$

Sánchez, M. M. I. (2000). Características y apreciaciones generales de los métodos de medida y estimación de la evapotranspiración. Revista de Geografía Norte Grande, 27, 27-36.

Senthelas, P. C., Gillespie, T. J., \& Santos, E. A. (2010). Evaluation of FAO Penman-Monteith and alternative methods for estimating reference evapotranspiration with missing data in Southern Ontario, Canada. Agricultural Water Management, 97, 635-644. DOI: 10.1016/j.agwat.2009.12.001

Tagliaferre, C., Santos, L. C., Santos, I. J., Santos, T. J., Rocha, F.A., \& Guimarães, D. U. (2012). Estimativa da evapotranspiração de referência com uso do irrigâmetro em Vitória da Conquista-BA. Irriga, Botucatu, 17(1), 28-38.

Tyagi, N. K., Sharma, D. K., \& Luthra, S. K. (2000). Determination of evapotranspiration and crop coefficients of rice and sunflower with Iysimeter. Agricultural Water Management, 45, 41-54.

Valencia, J. M., García, C. E., \& Montero, D. (2017). Anomalías de vegetación asociadas con el fenómeno del ENOS en el valle geográfico del río Cauca, Colombia. Revista de Teledetección, 50, 89-99. Recuperado de https://doi.org/10.4995/raet.2017.7715

Vicente-Serrano, S. M. , Azorín-Molina, C., Sánchez-Lorenzo, A., Revuelto, J., López-Moreno, J. I., González-Hidalgo, J. C., Moran-Tejada, E., \& 
Espejo, F. (2014). Reference evapotranspiration variability and trends in Spain, 1961-2011. Global and Planetary Change, 121, 26-40. Recuperado de http://dx.doi.org/10.1016/j.gloplacha.2014.06.005

Xu, C. Y., \& Singh, V. P. (2001). Evaluation and generalization of temperature-based equations for calculating evaporation. Hydrological Processes, 15, 305-319.

Yao, M. H., Li, M. H., Juan, J. Y., Hsia Y. J., Lee P. H., \& Shen, Y. (2017). Mapping reference evapotranspiration from meteorological satellite data and applications. Terrestrial Atmospheric and Oceanic Sciences, 28, 501-515. DOI: 10.3319/TAO.2016.11.15.01 